PALEO

Revue d'archéologie préhistorique

$20 \mid 2008$

Spécial table ronde ( $2^{\mathrm{e}}$ partie) : Le Gravettien : entités régionales d'une paléoculture européenne, Les Eyzies, juillet 2004

\title{
Les « Périgordiens » en Quercy : l'exemple du gisement des Fieux
}

"Perigordian" people in Quercy: the example of the site of Les Fieux.

\section{Patricia Guillermin}

\section{OpenEdition}

Journals

Édition électronique

URL : http://journals.openedition.org/paleo/1617

DOI : $10.4000 /$ paleo. 1617

ISSN : 2101-0420

Éditeur

SAMRA

Édition imprimée

Date de publication : 1 décembre 2008

Pagination : 357-372

ISSN : $1145-3370$

Référence électronique

Patricia Guillermin, «Les «Périgordiens » en Quercy : l'exemple du gisement des Fieux », PALEO [En

ligne], 20 | 2008, mis en ligne le 12 mai 2011, consulté le 07 juillet 2020. URL : http://

journals.openedition.org/paleo/1617 ; DOI : https://doi.org/10.4000/paleo.1617

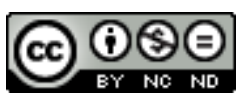

PALEO est mis à disposition selon les termes de la licence Creative Commons Attribution - Pas d'Utilisation Commerciale - Pas de Modification 4.0 International. 


\title{
LES « PÉRIGORDIENS » EN QUERCY : L'EXEMPLE DU GISEMENT DES FIEUX
}

\author{
Patricia GUILLERMIN ${ }^{(1)}$
}

\begin{abstract}
Résumé : L'occupation gravettienne du Quercy est aujourd'hui étudiée avec l'intention d'élucider les modalités d'exploitation de cet espace, en lien étroit avec son voisin immédiat, le Périgord. Cette démarche rejoint la question du polymorphisme d'ensembles gravettiens, à travers notamment le prisme de la fonctionnalité des sites. L'exemple de la couche $\mathrm{E}$ du gisement des Fieux illustre pleinement cette problématique. Son étude révèle la nécessité de replacer l'ensemble lithique dans son contexte environnemental, celui d'une occupation au sein d'un territoire parcouru et exploité de manière planifiée. Certains groupes gravettiens témoignent ainsi de fortes capacités d'adaptation, cherchant à optimiser les ressources disponibles en fonction de leurs objectifs immédiats ou différés.
\end{abstract}

Mots-Clés : Quercy, Gravettien, technologie lithique, polymorphisme, mobilité, spécialisation, stratégie économique.

Abstract: "Perigordian" people in Quercy: the example of the site of Les Fieux. The gravettian occupation of Quercy is today studied to elucidate its exploitation modalities, in close relation to neighbouring Périgord. This process comes to the question of the gravettian industries polymorphism, in particular through the prism of functionnality of sites. The example of layer $E$, belonging to the site of Les Fieux fully illustrates this problem. Its study shows the necessity to put back the lithic assemblage in its environnemental context, the one of an occupation within a territory covered and exploited according to a planification. Some gravettian groups thus show great capacities of adaptation, aiming at the optimization of avalaible ressources according to their immediate or future objectives.

Key-words: Quercy, Gravettian, lithic technology, polymorphism, mobility, specialization, economic strategy.

\section{INTRODUCTION}

Davantage qu'une culture homogène dans le temps comme dans l'espace, le Gravettien est aujourd'hui considéré comme un techno-complexe regroupant une mosaïque d'expressions culturelles. Même si de grands caractères communs rapprochent les industries gravettiennes, ce sont ces différences qui sont aujourd'hui analysées avec attention, afin de tenter d'interpréter la signification d'une telle variabilité des comportements techniques. En effet, ne serait-ce qu'à l'échelle du sud-ouest de la France, les ensembles gravettiens sont souvent qualifiés de polymorphes et difficiles à replacer les uns par rapport aux autres dans un schéma d'ensemble cohérent. En définitive, avant d'élaborer une problématique sur le Gravettien, il est nécessaire de bien considérer l'échelle à laquelle on se place : la détection de caractères communs peut en effet être opérée sur de grandes échelles géographiques et chronologiques, mais cette démarche conduit obligatoirement à minimiser les différences. Réfléchir à la signification de ces dernières nécessite peut-être de raisonner à moindre échelle géographique, afin de bien évaluer la part du milieu parmi les facteurs possibles de variabilité. Ainsi, en première approche, la recherche de l'interprétation du polymorphisme doit être entreprise au sein d'un espace relativement restreint pouvant correspondre à l'étendue d'un territoire paléolithique.

En liaison avec le travail que nous menons à plus large échelle sur les derniers temps du Gravettien, nous avons choisi de tester la pertinence de cette démarche en nous basant sur le Quercy².

(1) Université de Toulouse-Le Mirail, UMR 5608, TRACES, maison de la Recherche, 5 allées Antonio Machado, 31058 Toulouse Cedex 9 - patriciaguillermin@yahoo.fr

(2) Ce travail est réalisé dans le cadre d'une Action Commune de Recherche menée sur l'occupation paléolithique de cette région, coordonnée par $M$. Jarry. 
Pour l'instant, notre travail a concerné essentiellement la couche E du gisement des Fieux (Guillermin 2004 et 2006). Nous exposerons donc les pistes de réflexion que nous a amenées cette étude après avoir présenté rapidement les données disponibles sur l'occupation gravettienne du Quercy de manière générale.

\section{1 - L'ESPACE QUERCINOIS}

Situé entre le Bassin aquitain et le Massif central, le Quercy se caractérise par la présence des grands causses, vastes plateaux calcaires du Jurassique moyen et supérieur. Ils sont traversés par des vallées encaissées bordées de grottes et d'abris, témoins d'un réseau karstique très important. Toutes ces vallées sont autant d'axes de circulation potentiels, ainsi la Dordogne permet de rejoindre le Périgord ou le Bassin de Brive, tandis que le Lot rencontre le Haut-Agenais. Enfin, la vallée de l'Aveyron, à l'extrême sud, peut constituer un lien entre cet espace nord-aquitain, le Languedoc et les Pyrénées.

Aux limites des causses du Jurassique, au nord-ouest, les paysages de la Bouriane marquent la transition vers les plateaux calcaires du Crétacé supérieur du Périgord, couverts d'argile et de sables paléogènes. Au sud-est, le BasQuercy rejoint le Haut-Agenais, découvrant des plateaux et des " serres " de calcaire lacustres découpés par des vallées creusées dans des molasses surtout oligocènes.

\section{2 - FENÊTRE SUR L'OCCUPATION GRAVETTIENNE DU QUERCY (fig.1)}

\section{1 - Quelles données ?}

Comparés à la richesse du Périgord, les gisements du Quercy offrent, à première vue, peu de données exploitables. Certains ont été fouillés anciennement et présentent des assemblages mélangés, c'est plus particulièrement le cas des gisements proches de la vallée de l'Aveyron : la grotte du Nid d'Aigle, le Cuzoulet mais aussi le Roc de Cavart. D'autres informations ont été recueillies à partir du tamisage des déblais comme pour la grotte du Rouzet ou l'abri du Syndic. Quelques ramassages de surface ont livré des indices explicites d'occupation, notamment aux stations de Mirande et de Peyro-Lebado 2. Les informations sont donc considérablement tronquées pour ces gisements. A contrario, les grottes des Escabasses et de Pégourié ont fait l'objet d'investigations plus récentes, mais les indices récoltés en fouilles sont très faibles. Cependant, d'autres sites, fouillés plus récemment, présentent des stratigraphies riches d'informations : ce sont les gisements de la Bergerie, des Peyrugues et des Fieux situés au cœur du Quercy, ainsi que les abris de Roc-deCombe et des Battuts bordant les frontières quercinoises.

\section{2 - Des environnements diversifiés}

La carte de répartition des différents sites révèle des occupations diversifiées de l'espace. Certains gisements sont

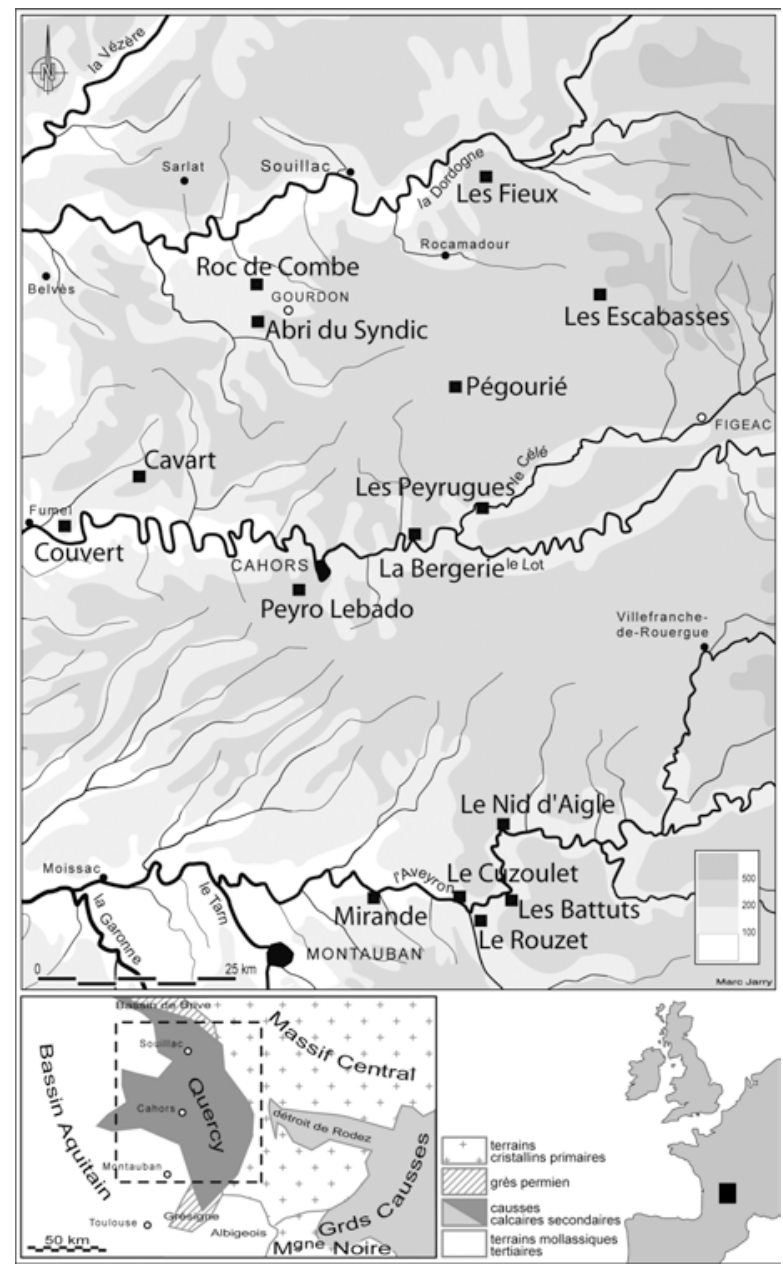

Figure 1 - Carte de répartition des différents gisements gravettiens du Quercy (fond de carte M. Jarry).

Figure 1 - Location of the differents gravettian sites in Quercy (map of M. Jarry).

placés au cœur du Quercy, dans la région des grands causses. Comme la majeure partie des occupations du Paléolithique supérieur de cette région, ils peuvent être situés dans les vallées, à proximité des grands cours d'eau (les Peyrugues, la Bergerie), tandis que d'autres témoignent de l'occupation des plateaux (les Fieux).

Plus de la moitié des gisements cités se trouvent néanmoins aux frontières du Quercy. Si de vastes espaces restent ainsi vides, il est impossible de l'interpréter comme une occupation différentielle du Quercy, les prospections faisant vraisemblablement défaut dans ce secteur.

\section{3 - Un espace occupé durant tout le Gravettien}

Malgré la faiblesse des informations livrées par la plupart des gisements, les indices permettent de conclure à une grande couverture chronologique des données quercinoises. Le Quercy a manifestement été un espace inscrit sur une très longue durée dans la géographie des Gravettiens du nord de l'Aquitaine. 


\subsection{1 - Le Haut-Quercy}

Dans le Haut-Quercy, certains sites sont attribués au Gravettien moyen par la présence de burins de Noailles, ces derniers semblant demeurer de bons marqueurs chronologiques. C'est le cas notamment de la grotte de Pégourié (Caniac-du-Causse, Lot) et de Peyro-Lebado 2 (SaintVincent-Rive d'Olt, Lot) (Clottes 1971 ; Séronie-Vivien 1995).

Les indices gravettiens retrouvés dans les déblais de l'abri du Syndic (Saint-Cirq-Madelon, Lot) ne permettent pas de conclure sur le faciès concerné, nous pouvons juste noter la présence d'une pointe de La Font-Robert (Lorblanchet et Genot 1972).

M. Lorblanchet a effectué un sondage de deux mètres carrés à la grotte des Escabasses (Thémines, Lot) (Lorblanchet 1966). II distingue des indices d'occupation gravettienne, sus-jacents à un niveau solutréen. Nous émettons des réserves sur cette affirmation : d'une part l'aspect taphonomique n'a pas été abordé (la faune de la couche contenant les deux niveaux est dominée par le lapin avec un état de conservation « étonnant »...). De plus, les éléments gravettiens représentés par l'auteur ne sont pas caractéristiques, enfin : "les éléments typiques protosolutréens proviennent du carré $A$ et les gravettiens du carré $B$ » (Lorblanchet 1966 - p. 276). II semble donc difficile d'attribuer cet ensemble à un faciès chronologique précis.

D'autres gisements ont connu une succession d'occupations gravettiennes observées en stratigraphie :

Le site de la Bergerie (Saint-Géry, Lot) possède un niveau à pointes de La gravette seules sous un niveau à burins de Noailles, il s'agit donc vraisemblablement d'un Gravettien ancien (Clottes et al. 1990 ; Peyre 1988).

Le Gravettien récent se retrouve dans la stratigraphie de Roc-de-Combe (Payrignac, Lot) (couche 1), au-dessus de deux niveaux à burins de Noailles (couches 2 et 3 ) et d'un niveau à gravettes (couche 4). La couche 1 a été attribuée au «Périgordien évolué » d'après sa position stratigraphique au-dessus de niveaux appartenant au Gravettien moyen (Bordes et Labrot 1967 ; Sonneville-Bordes 2002). Cela peut être effectivement le cas mais l'intégrité du niveau concerné doit être remise en question, d'une part par la présence de pointes de La Font-Robert, de burins de Noailles ainsi que d'éléments aurignaciens, d'autre part, d'après la description faite du niveau (les auteurs parlant de fosse creusée par les paléolithiques traversant les couches 2 à 5).

La stratigraphie des Peyrugues (Orniac, Lot), gisement fouillé récemment (Allard 1996 ; Allard et al. 1997), révèle la succession de haut en bas d'un niveau attribué au Gravettien final (couche 18), de plusieurs niveaux récents (couches 20, 20a, 21b, 21d, 22) et d'au moins deux niveaux attribués au Gravettien moyen à burins de Noailles (couches 28 et 30 ). La présence de Gravettien final (ou "Protomagdalénien ") permet d'associer les Peyrugues aux quatre autres seuls sites attribués à ce faciès que sont l'Abri Pataud, Laugerie-Haute (Les Eyzies-de-Tayac, Dordogne) le
Blot (Cerzat, Haute-Loire) et d'une manière moins certaine Rabier (Lanquais, Dordogne) (Bordes 1978 ; Bordes et Sonneville-Bordes 1966 ; Bricker 1995 ; Clay 1968 ; Delporte 1972 ; Lorin 1996 ; Morala 1990, entre autres).

\subsection{2 - La vallée de l'Aveyron et ses alentours}

En marge du Quercy, dans la vallée de l'Aveyron, les indices d'occupation gravettienne sont présents mais peu nombreux, ce fait pouvant être dû à un manque de prospection.

Le gisement le plus important est celui des Battuts (Penne, Tarn), fouillé par J.-F. Alaux de 1967 à 1970 (Alaux 1967, 1969 et 1973). La stratigraphie comprend quatre niveaux gravettiens, de bas en haut : un niveau à 'éléments tronqués' (c. 5), un niveau riche en burins de Noailles (c.7) et deux niveaux attribués au Périgordien évolué (c.9 et 12). Toutefois, nous avons mis en avant les problèmes liés à la validité de la stratigraphie de ce site (Guillermin 2003).

Ainsi, la couche 12 reste introuvable au sein de l'assemblage, peut-être a-t-elle été regroupée avec la couche 9 , cette appellation concernant des niveaux différents suivant les années. En outre, l'assemblage de cette couche ne comporte que 47 outils, parmi lesquels se trouvent 21 burins de Noailles, il serait donc à rapprocher d'un faciès à burins de Noailles.

La couche 7 a été observée dans une autre partie du site, au fond de l'abri, sans positionnement stratigraphique explicite avec les couches 5 et 9-12. II s'agit d'un niveau très riche en burins de Noailles. Deux pointes de La FontRobert ont été trouvées et quelques burins du Raysse sont présents, mais peu nombreux, atypiques et provenant essentiellement des déblais.

La série de la couche 5 présente peu de caractères distinctifs si ce n'est sa position stratigraphique et le fait qu'elle comporte des éléments tronqués ainsi que 3 burins de Noailles au sein de 50 outils. Une première étude très ponctuelle réalisée sur cet ensemble dans le cadre d'un mémoire de maîtrise nous avait amenée à proposer un rapprochement au faciès ancien (Guillermin 2003). Néanmoins, une comparaison critique des ensembles C5-C9 réalisée plus récemment (Guillermin, étude en cours) ne nous donne aucun élément distinctif entre eux. Associons à cette observation le fait que le marquage des pièces par carré ne coïncide absolument pas avec l'évolution logique de la succession des couches. Cette remarque concerne également l'ensemble aurignacien de la couche 3 sous-jacente, qui comporte par ailleurs des éléments gravettiens.

Nous concluons à un remaniement des séries archéologiques lors du conditionnement qui, en l'absence des carnets de fouilles qui permettraient de réattribuer les pièces aux différentes couches, décourage toute approche rigoureuse.

D'autres gisements sont cités par B. Pajot dans son inventaire des occupations du Paléolithique supérieur du bassin de l'Aveyron (Pajot 1969). 
II s'agit notamment des grottes du Nid d'Aigle (SaintAntonin, Tarn-et-Garonne) mais les assemblages sont issus de fouilles anciennes et sont mélangés ; aucune stratigraphie n'a été relevée. La présence de quelques éléments gravettiens, notamment un burin de Noailles, peut être notée.

Le matériel du gisement du Cuzoulet (Bruniquel, Tarn-etGaronne) est également mélangé, révélant néanmoins des indices d'occupation gravettiennes, dont une pointe de La Font-Robert.

La station de Mirande (Nègrepelisse, Tarn-et-Garonne) aurait livré des éléments gravettiens mais le doute subsiste.

Un peu plus au sud, la grotte du Rouzet (Larroque, Tarn) a fait l'objet d'une reprise de travaux par J-F Alaux qui a tamisé les déblais. Cela lui a permis de confirmer l'existence d'au moins une occupation gravettienne à burins de Noailles (Alaux 1965)

\subsection{3 - Le Haut-Agenais}

Au sud-ouest du Quercy, le Haut-Agenais connaît de nombreux témoins d'occupations gravettiennes. Cette région a bénéficié notamment des travaux de J-M. Le Tensorer et d'A. Morala (Le Tensorer 1981 ; Morala 1984 et 1989). Certains sites, situés en marge de cette région, sont cités dans les inventaires du Quercy, il s'agit des abris de Couvert et de Roc de Cavart.

Des burins de Noailles sont présents parmi les quelques vestiges qui ont été retrouvés à l'abri de Couvert (Soturac, Lot). Ces marqueurs chronologiques nous permettent de parler d'indices d'occupation attribuables au Gravettien moyen (Clottes 1971 et 1979 ; Morala 1984 ; Guillermin et Morala, à paraître).

L'abri de Roc de Cavart (Montcabrier, Lot), fouillé par L. Coulonges en 1927, présente, sous une couche solutréenne, un niveau gravettien interprété comme «Périgordien évolué» (Coulonges 1949 ; Le Tensorer 1981 ; Morala 1984). II s'agit de fouilles anciennes et l'effectif de la série est relativement réduit (320 artéfacts lithiques dont 252 outils). La présence de pointes à face plane solutréennes dans l'assemblage illustre son hétérogénéité. Il semble que le niveau ait subi des contaminations dont le degré peut se révéler élevé : en effet, le reste de l'outillage semble très divers, cette diversité se manifestant même au niveau des supports de lamelles à dos (la pointe à face plane révélant une contamination du niveau sus-jacent, certaines de ces armatures pourraient être solutréennes). Par ailleurs, les deux éléments qualifiés de burins de Noailles ne sont pas typiques puisqu'ils ne possèdent pas de coches d'arrêt. II est donc difficile de conclure sur l'occupation de ce site. Aucun élément ne permet de l'attribuer à une phase du Gravettien en particulier (Guillermin et Morala, à paraître).

Ainsi, la reprise des données concernant l'occupation gravettienne du Quercy révèle, d'une part, un espace connu et occupé pendant toute la période concernée et, d'autre part, une exploitation de la diversité environnementale de cet espace, entre les différentes vallées et le causse.
Si nous tentons à présent de mener une réflexion sur les modalités d'exploitation de l'espace quercinois par les gravettiens, il est nécessaire d'approfondir l'étude des ensembles archéologiques. Certains sites sont en mesure de constituer un corpus d'étude satisfaisant pour approfondir nos investigations. C'est le cas notamment du gisement des Fieux, dont l'étude de la couche $\mathrm{E}$ a fait l'objet d'un mémoire de DEA (Guillermin 2004).

\section{3 - LE GISEMENT DES FIEUX : UNE OCCUPATION PARTICULIÈRE DU CAUSSE ?}

\section{1 - Présentation du gisement}

Situé sur le causse de Gramat, le gisement des Fieux est fouillé par F. Champagne de 1967 à 1991 (Champagne 1977 et 1987 ; Champagne et Jaubert 1979 et 1986 ; Champagne et al. 1990 et 1996) (fig. 2). Le secteur central du site est constitué d'une dépression issue de l'effondrement d'un boyau karstique. Des porches s'ouvrent aux extrémités est et ouest de cette surface, révélant le prolongement du réseau. L'un d'eux, à l'ouest, offre l'accès à une grotte ornée comportant notamment des mains négatives (Glory 1965 ; Lorblanchet 1984a et b ; Nougier et Barrière 1965 entre autres).

La stratigraphie de ce gisement, exprimée différemment suivant les secteurs, révèle des témoins d'occupation couvrant un large éventail chronologique, entre Moustérien et Néolithique (fig. 3). Le Gravettien est présent dans tous les secteurs. A l'Ouest, les indices sont faibles et aucun niveau n'a été individualisé. La présence d'une pointe de La Font-Robert, de pointes de La gravette ainsi que de burins du Raysse est à signaler.

A l'Est, F. Champagne présente une stratigraphie où la couche $E$, principal ensemble gravettien, se superposerait à un niveau solutréen $(\mathrm{F} 1 \mathrm{a})$, à deux niveaux gravettiens (F1b et F1c) et à un niveau aurignacien (F2) (fig.4). L'un des deux premiers niveaux gravettiens, F1c, est daté à 23900 \pm 330 BP (Gif 6303). Cependant les effectifs des deux ensembles sont très faibles (autour d'une cinquantaine d'outils) et leur faciès n'est pas caractérisé avec précision.

De plus, l'assemblage de la couche F1a regroupe des éléments gravettiens et quelques outils solutréens qui ont été trouvés lors de l'extension de la fouille vers le porche est, à un endroit où la couche E n'est plus présente (fig. 5). Ceci remet en cause l'idée d'une superposition de la couche $E$ à un niveau solutréen. En réalité, l'ensemble de la stratigraphie de ce porche doit être revue, nous incitant à limiter, dans un premier temps, l'étude de la couche $\mathrm{E}$ au secteur central.

\section{2 - La couche E : un assemblage gravettien original}

La couche E est constituée d'un sédiment limono-argilosableux, considéré jusqu'à présent comme un apport éolien et comblant la large dépression occupant la partie centrale du gisement. L'acidité de ce sédiment n'a pas permis la conservation de l'os. 

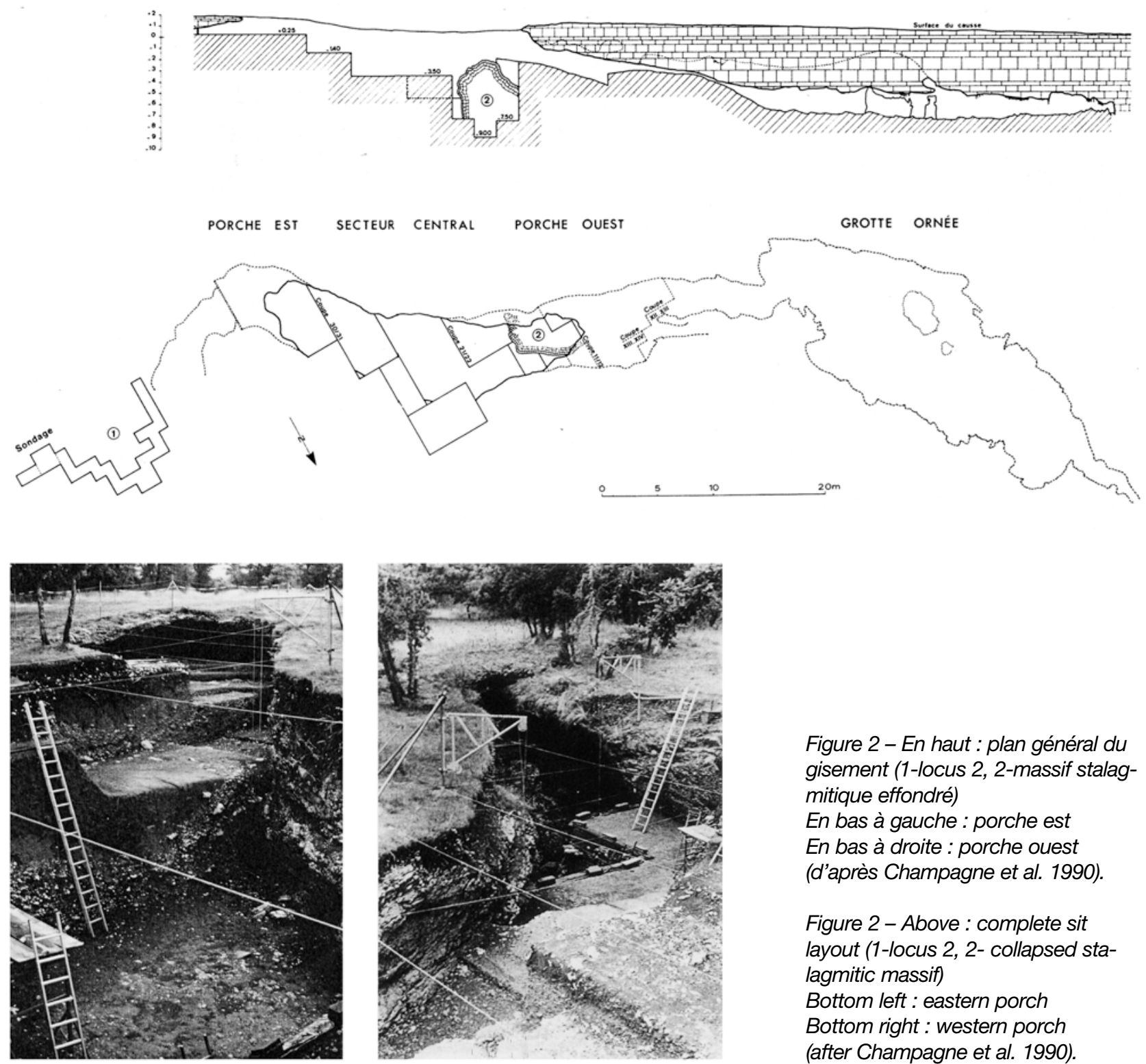

Figure 2 - En haut : plan général du gisement (1-locus 2, 2-massif stalagmitique effondré)

En bas à gauche : porche est

En bas à droite : porche ouest (d'après Champagne et al. 1990).

Figure 2 - Above : complete sit layout (1-locus 2, 2- collapsed stalagmitic massif)

Bottom left : eastern porch

Bottom right : western porch (after Champagne et al. 1990).

Cet apport sédimentaire, très différent des dépôts cryoclastiques sous-jacents, avait été interprété par F. Champagne comme imputable au changement climatique post-würmien. Cette interprétation, associée à la présence de niveaux mésolithiques sus-jacents, avait amené F. Champagne à attribuer la série à de l'Epipaléolithique. II est, par la suite, revenu sur cette assertion, proposant l'hypothèse d'une attribution au Protomagdalénien ou Gravettien final. Cette nouvelle orientation fut probablement aiguillée par la présence importante dans l'outillage de lamelles à dos tronquées, parfois bitronquées (tabl. 1) (Champagne 1986-1987 ; Champagne et al. 1990).

L'observation de l'assemblage confirme l'attribution au Gravettien, notamment par la présence de pointes et micropointes de La Gravette issues de schémas opératoires appartenant sans équivoque à cette culture (tab. 1, fig. 6 et 11b).
A ceci s'ajoutent des éléments du faciès gravettien moyen dit «Rayssien » tels qu'ils ont été caractérisés par L. Klaric : des burins du Raysse, une lamelle de La Picardie, des nucléus laminaires portant des aménagements rappelant la méthode du Raysse et des lames à facettage latéralisé oblique (tab.1 et fig.7). Cependant, l'association de ces éléments aux pointes de La Gravette et aux schémas producteurs de leurs supports ne correspond pas au faciès rayssien tel qu'il a été défini par $L$. Klaric à partir des sites de la Picardie et d'Arcy-sur-Cure (Klaric 2003 ; Klaric et al. 2002). Une évaluation taphonomique permet néanmoins d'envisager la relative homogénéité de l'assemblage (Guillermin 2004), entérinant cette association.

La présence des burins du Raysse nous invite alors à attribuer la couche E au Gravettien moyen. Cependant, les sites du sud-ouest de la France attribués à ce faciès, à 


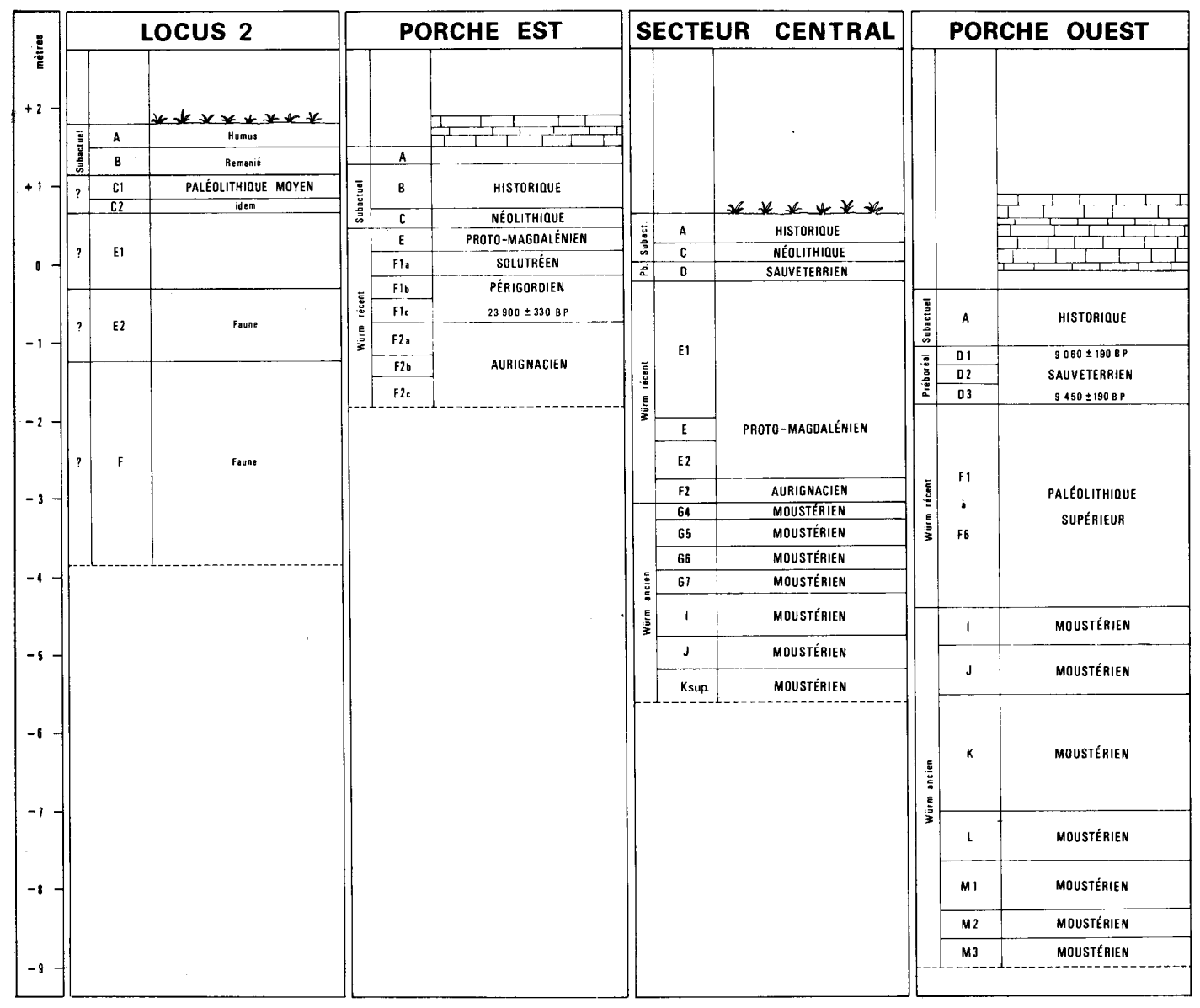

Figure 3 - Les différentes stratigraphies du gisement par secteur (d'après Champagne et al. 1990).

Figure 3 - Stratigraphy of the site described per area (after Champagne et al. 1990)
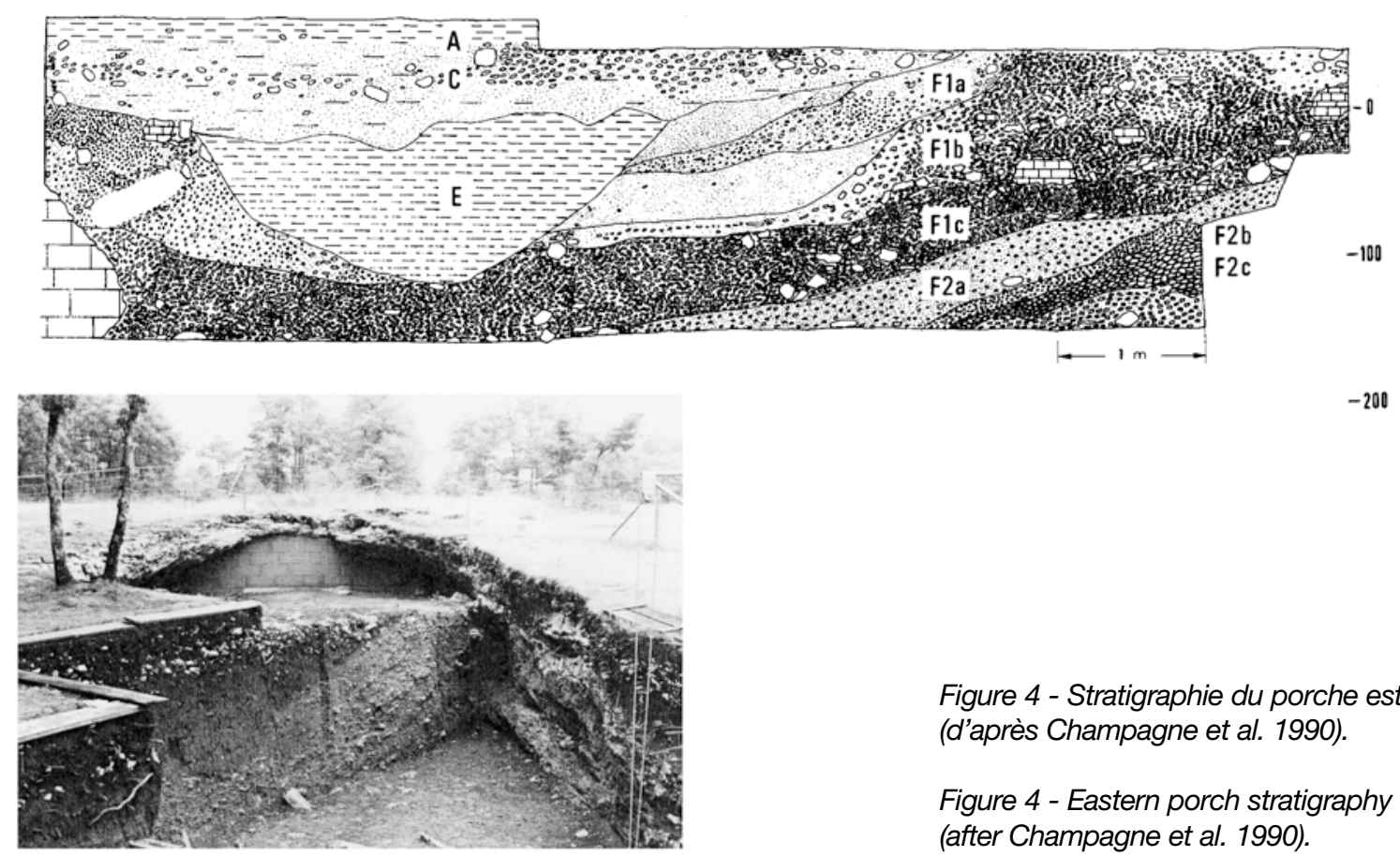

Figure 4 - Stratigraphie du porche est (d'après Champagne et al. 1990).

Figure 4 - Eastern porch stratigraphy (after Champagne et al. 1990). 

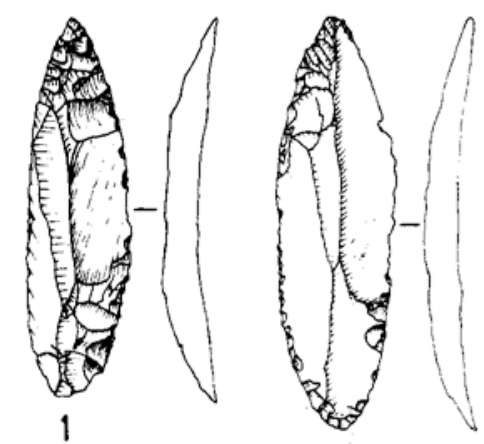

2
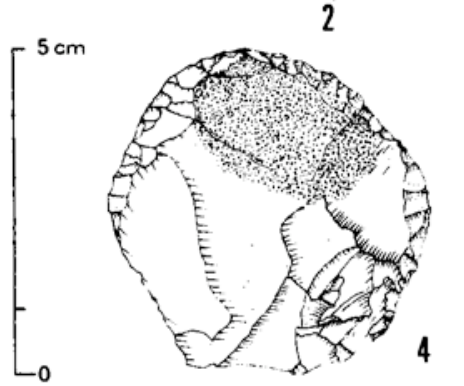

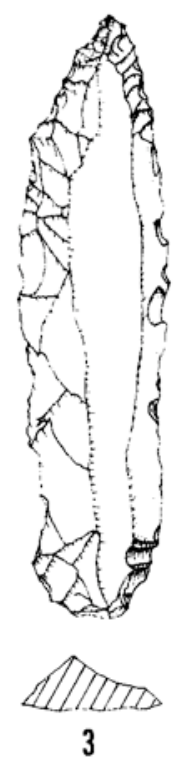

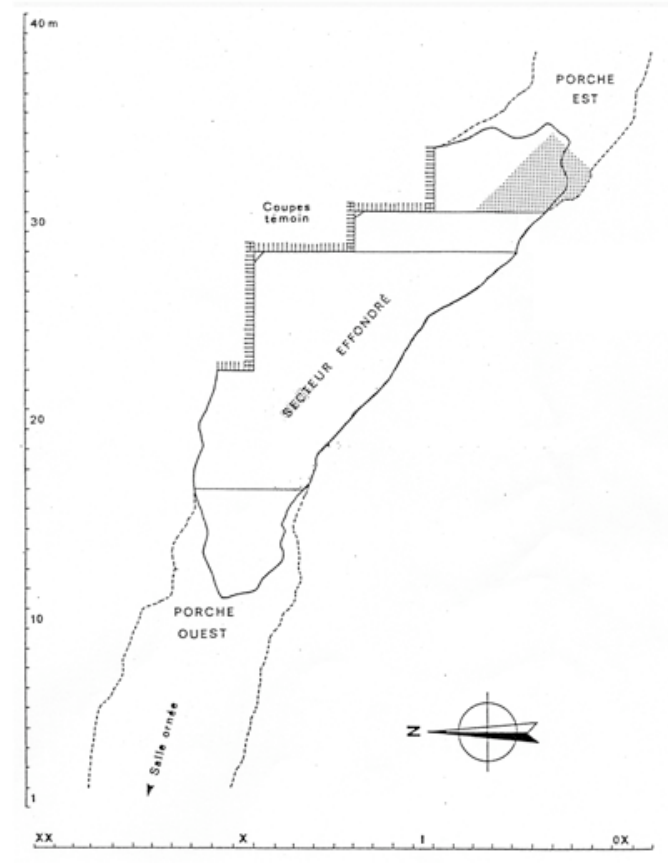

Figure 5 - A gauche : les outils solutréens de la couche F1a; à droite : la zone hachurée correspond à l'extension des fouilles pour dégager le porche est, les outils solutréens ont été trouvés à cet endroitr alors que la couche E n'est plus présente.

Figure 5 - Left : solutrean tools from the F1a; Right : the hatched area corresponds to the excavation extension to clear the eastern porch. The solutrean tools were found in this area where the $E$ layer is not in place anymore.

l'exemple du gisement du Flageolet 1 (Lucas 2000 ; Rigaud 1982), de Solvieux (Sackett 1999) ou de la couche 4 de l'Abri Pataud (Pottier 2005), comportent également un autre marqueur culturel fort : le burin de Noailles. Cet élément est totalement absent du gisement des Fieux. Ce phénomène peut être interprété comme résultant d'un caractère plus évolué de la couche E, la rapprochant du Gravettien récent.

Ainsi, l'association d'éléments rayssiens au système producteur de pointes et micropointes de La Gravette sans la présence des burins de Noailles peut être interprétée comme l'expression d'un faciès chronologique méconnu (Guillermin 2006). C'est un des caractères originaux de la couche E mais ce n'est pas le seul : une autre particularité est la diversité des armatures dominées -hormis les lamelles à dos- par des lamelles à dos tronquées (fig. 8).

Cet ensemble demeure donc original et continue à soulever des questions. II suscite une interrogation sur l'interprétation de ce phénomène dans l'étude typo-technologique du matériel. Outre l'aspect chronologique déjà évoqué, plusieurs questions peuvent être abordées, notamment celle de la fonction du site et de son éventuelle spécialisation. Nous pouvons également envisager d'être face à une spécialité régionale, autrement dit un faciès quercinois. Ces différents aspects reprennent, une fois encore, les hypothèses déjà énoncées par les préhistoriens qui se sont retrouvés face à l'interprétation du polymorphisme gravettien (Bordes
1968 ; Delporte 1983 ; Laville et Rigaud 1973 ; Peyrony 1946 ; Rigaud 1983 et 1985 ; Sonneville-Bordes 1966).

Pour notre part, nous allons aborder ces questions en réfléchissant à l'incidence des stratégies économiques d'exploitation des ressources minérales sur la constitution des ensembles lithiques.

3.3 - Des armatures nombreuses et diverses : des indices de spécialisation dans la chasse

L'assemblage lithique révèle une forte proportion d'armatures (près de $50 \%$ de l'outillage, fig. 9) portant, pour nombre d'entre elles, des fractures diagnostiques d'impact. La présence de pointes -avec ce type de dommage- peut être interprétée comme l'introduction, à l'intérieur de l'abri, de carcasses porteuses de ces éléments. Ainsi, même si l'os n'est pas conservé, plusieurs indices permettent d'envisager une activité cynégétique importante exécutée aux abords du site, nous amenant à l'hypothèse d'une spécialisation de l'occupation des Fieux par la chasse.

Les armatures sont diverses : certaines, minoritaires, sont sur support laminaire (pointes de La Gravette mais aussi des lames apointées portant des fractures d'impact), d'autres sont microlithiques - réalisées sur petites lames ou lamelles - (microgravettes, lamelles à dos dont la moitié sont tronquées, parfois bitronquées) (fig. 6 et 8). 

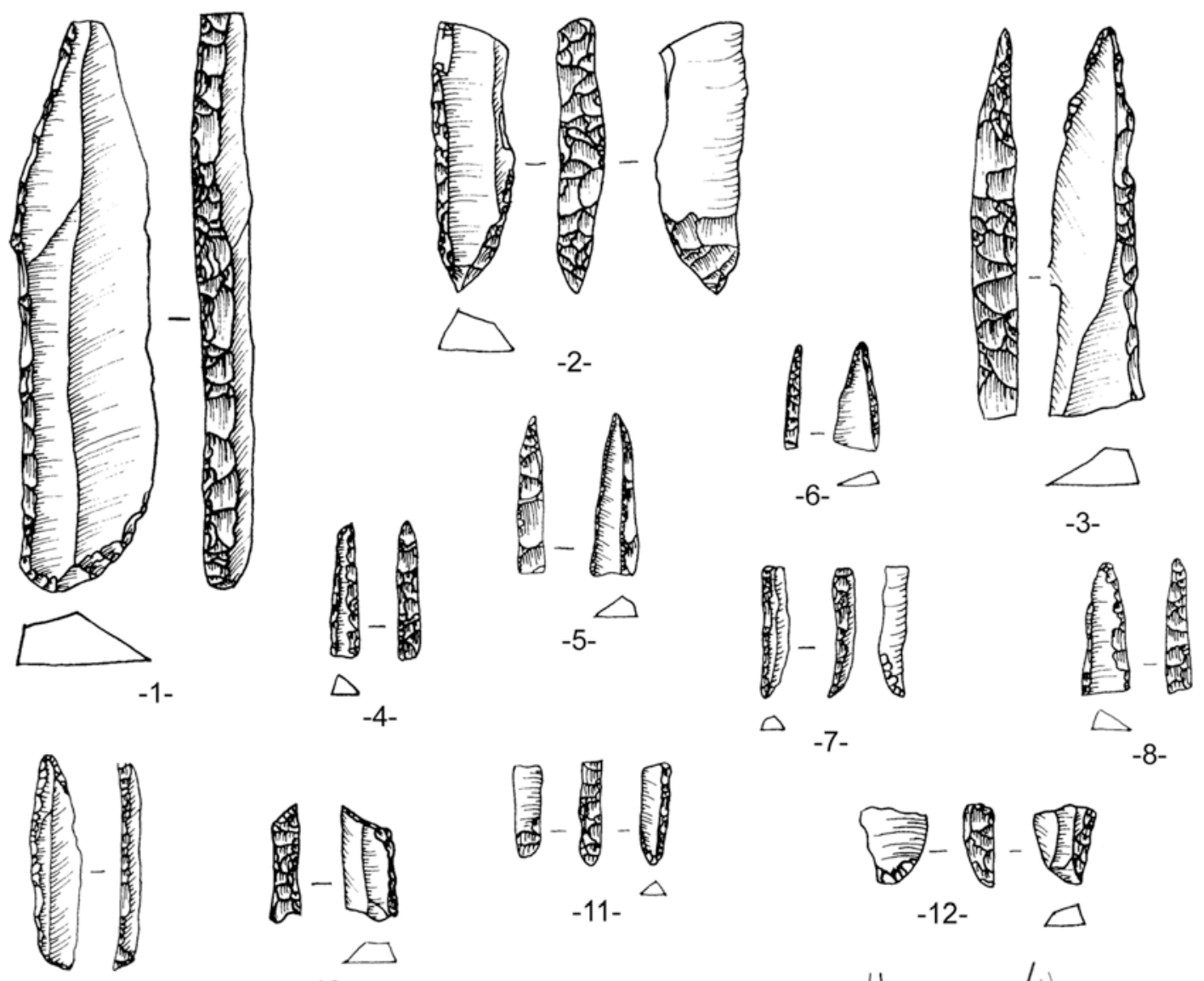

$\triangle$-7-
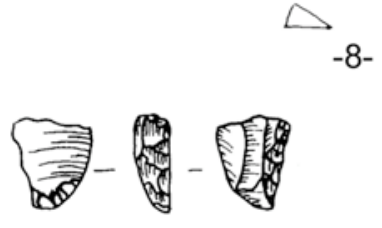

$-12-\square$

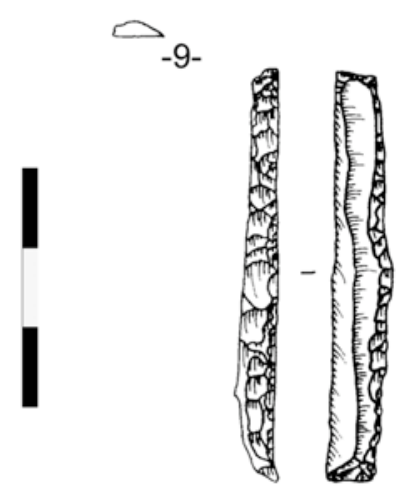

$-10-$
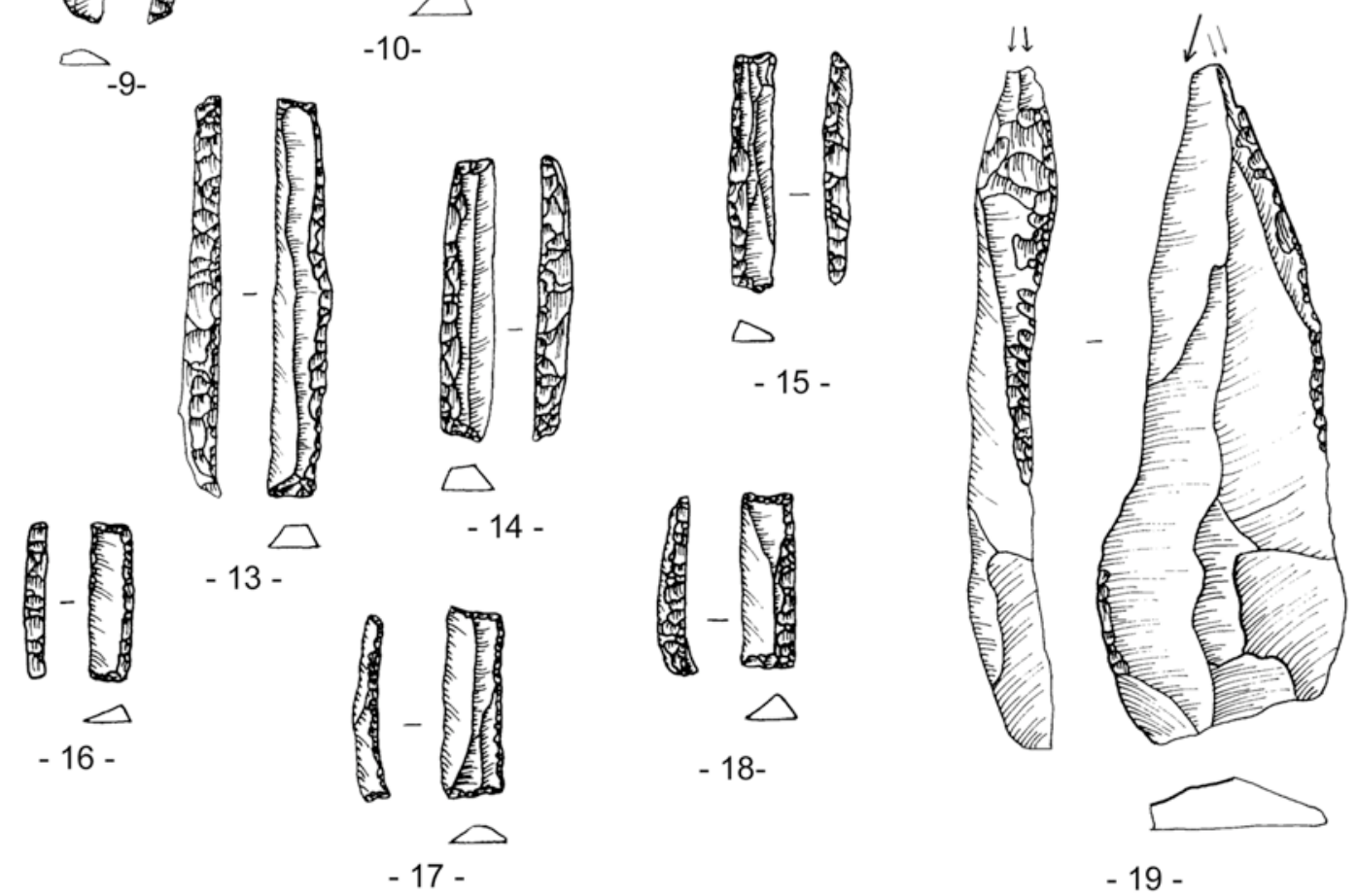

$-19-$

Figure 6 - Les armatures de la couche $E$ : pointes de La Gravette (1 à 3), microgravettes (4 à 12), lamelles à dos tronqué (13 à 18), lame apointée portant des fractures burinantes latérales (19).

Figure 6 - The projectile points of the E layer : Gravette's points (1 to 3), microgravettes (4 to 12), backed truncated bladelets (13 to 18), pointed blade showing traces of lateral hammering (19). 

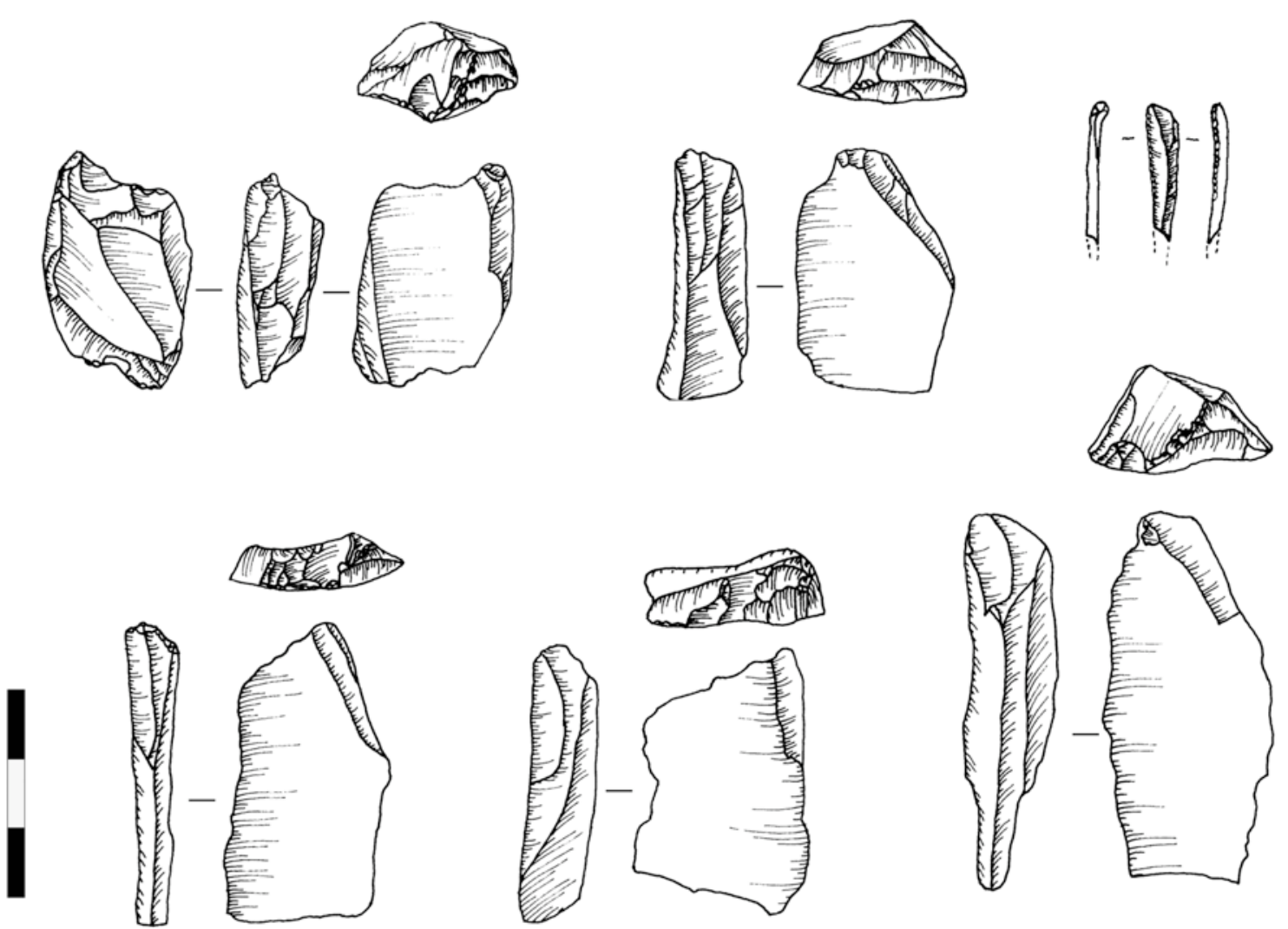

Figure 7 - Quelques éléments « rayssiens » de la couche $E$ : une lamelle de La Picardie et des burins du Raysse.

Figure 7 - Some « rayssiens » elements from the E layer : one Picardie's bladelet and several « burins du Raysse ».
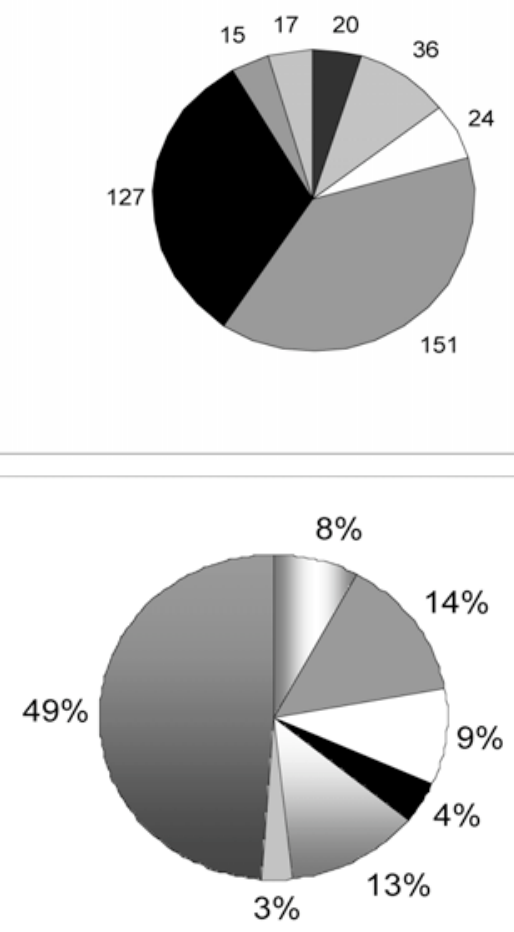

- gravettes

$\square$ microgravettes

$\square$ lames à dos

口 II à dos

- II à dos tronquées

घ II retouchées

- lames apointées
ㅁ grattoirs/perçoirs

๑ burins

$\square$ lames retouchées

- pièces esquillées

$\square$ éclats

ret/racloirs/enc./dentic.

$\square$ divers

a armatures
Figure 8 - Effectifs et proportions des différentes armatures de la couche $E$.

Figure 8 - Quantities and statistics of the various supports issued from the $E$ layer.

Figure 9 - Proportion des différents types d'outils au sein de l'industrie de la couche $E$.

Figure 9 - Porportion of the various tools-types issued from the $E$ layer. 


\begin{tabular}{|c|c|}
\hline catégorie typologique & effectif \\
\hline grattoir sur lame & 10 \\
\hline grattoir atypique & 14 \\
\hline grattoir double & 2 \\
\hline grattoir ogival & 1 \\
\hline grattoir sur lame retouchée & 16 \\
\hline grattoir sur éclat & 10 \\
\hline grattoir caréné atypique & 5 \\
\hline grattoir-burin & 5 \\
\hline perçoir atypique & 7 \\
\hline burin dièdre droit & 8 \\
\hline burin dièdre déjeté & 8 \\
\hline burin dièdre d'angle & 8 \\
\hline burin sur cassure & 22 \\
\hline burin dièdre multiple & 7 \\
\hline burin sur troncature droite & 4 \\
\hline burin sur troncature oblique & 10 \\
\hline burin sur troncature concave & 25 \\
\hline burin sur troncature convexe & 10 \\
\hline $\begin{array}{l}\text { burin sur troncature } \\
\text { transversale }\end{array}$ & 1 \\
\hline burin multiple mixte & 10 \\
\hline burin multiple mixte & 12 \\
\hline burin nucléiforme & 2 \\
\hline burin plan & 22 \\
\hline burin du Raysse & 23 \\
\hline pointe de la Gravette & 17 \\
\hline pointe de la Gravette atypique & 3 \\
\hline microgravette & 36 \\
\hline lame à dos & 24 \\
\hline lame à dos tronquée & 6 \\
\hline lame à troncature droite & 3 \\
\hline lame à troncature oblique & 11 \\
\hline lame à troncature concave & 9 \\
\hline lame à troncature convexe & 1 \\
\hline lame bitronquée & 2 \\
\hline lame à retouche à un bord & 19 \\
\hline lame à retouche à deux bords & 25 \\
\hline encoche & 25 \\
\hline denticulé & 9 \\
\hline pièce esquillée & 34 \\
\hline racloir & 4 \\
\hline lamelle tronquée & 6 \\
\hline lamelle à dos & 151 \\
\hline lamelle à dos tronquée & 102 \\
\hline lamelle à dos bitronquée & 25 \\
\hline lamelle à dos partiel & 4 \\
\hline lamelle retouchée & 9 \\
\hline lame apointée & 17 \\
\hline divers & 21 \\
\hline Total & 805 \\
\hline
\end{tabular}

Tableau 1 - Inventaire typologique d'après la liste type de $D$. de SonnevilleBordes et J. Perrot (Sonneville-Bordes et Perrot 1954, 1955 et 1956).

Table 1 - Typological inventory according to type-list written by $D$. de Sonneville-Bordes et J. Perrot (Sonneville-Bordes et Perrot 1954, 1955 et 1956).
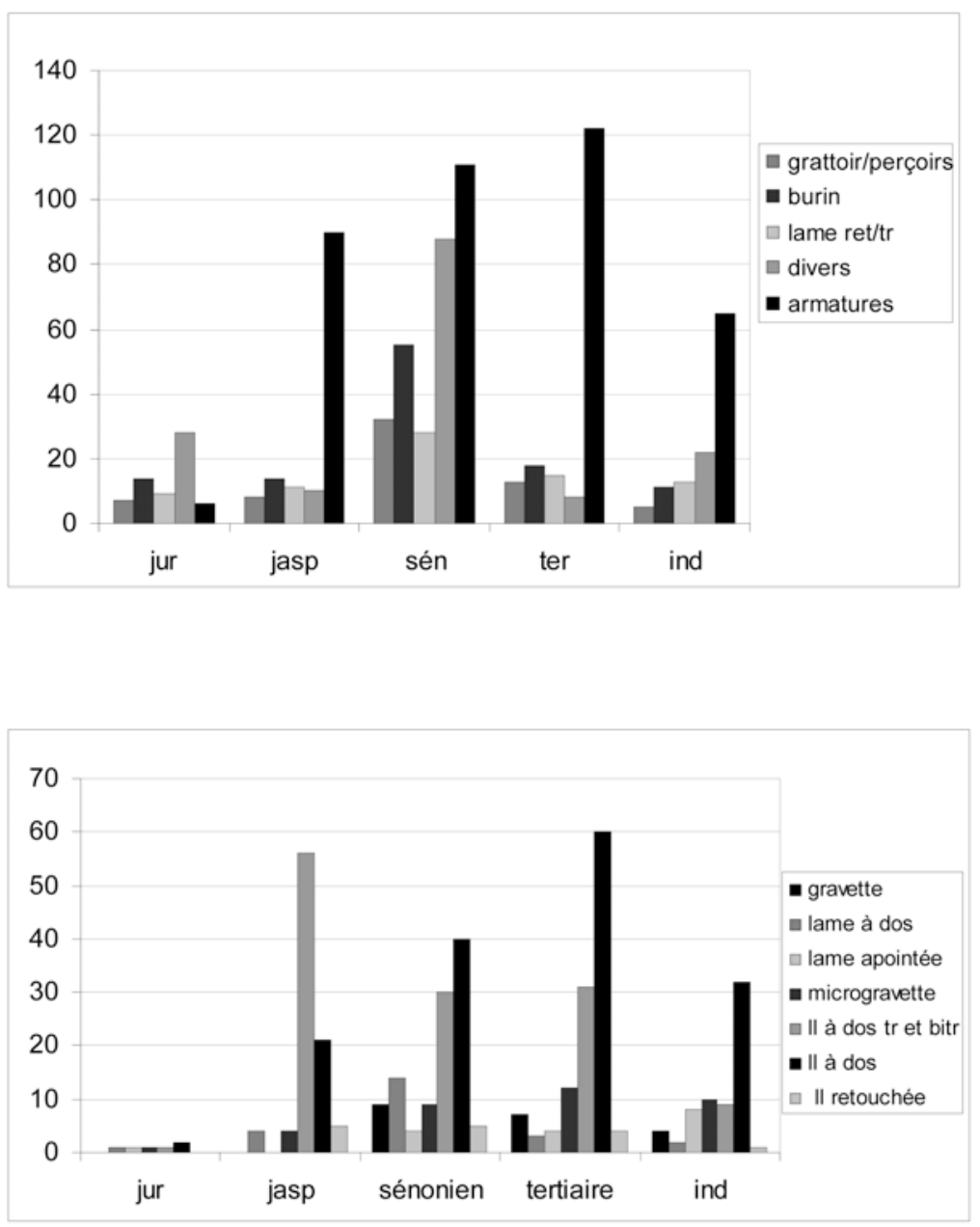

Figure 10 - En haut : répartition des types d'outils suivant les matières premières.

En bas : répartition des types d'armatures suivant les matières premières.

Figure 10 - Above : distribution of tool-types according to raw materials. Below : distribution of projectile points types according to raw materials. 
La question de la diversité des armatures au sein d'une occupation spécialisée reste alors posée : y a-t-il un lien entre cette diversité et la forte implication du groupe dans l'activité de chasse?

\section{4 - Fenêtre sur les stratégies économiques des occu- pants de la couche $E$}

Les supports de ces armatures constituent les différents objectifs du débitage observé sur le site. Son étude a révélé un traitement différentiel des matières premières, mettant l'accent sur l'aspect économique.

La caractérisation des différentes matières premières de la couche E est réalisée par P. Chalard (Chalard et al. 2006). Les matières premières locales (silex jaspéroïdes de I'Infralias, silex du Jurassique et quelques galets de silex tertiaire) sont majoritaires au sein du débitage. Les matières allochtones sont en grande majorité des silex sénoniens du Périgord, notamment du Campanien inférieur de la région de Belvès. Le silex maastrichtien de la région de Bergerac est représenté par quelques éléments. C'est le cas également des silex du Haut- Agenais mais aussi du silex dit « Grain de Mil » dont certains gîtes sont connus en Charente.

Le traitement différentiel des matières premières se révèle tout d'abord à travers les différentes proportions d'outils (fig. 10) : l'outillage à usage domestique en silex sénonien est en proportion plus importante que les armatures (64\% des outils) alors que les pourcentages sont inversés pour les silex jaspéroïdes et tertiaires. Par ailleurs, pratiquement aucune armature en silex du Jurassique n'est présente sur le gisement.

Ensuite, la répartition des divers types d'armatures par matière première (fig. 10) montre une production très ciblée des silex jaspéroïdes vers les lamelles à dos tronquées, tandis que la production en silex sénonien est beaucoup plus variée, concernant l'ensemble des armatures.

\subsection{1 - Une exploitation adaptée des ressources locales}

En réalité, l'orientation préférentielle dans la production en silex jaspéroïde dévoile une exploitation très adaptée à ce type de matière.

En effet, les lamelles à dos tronquées sont des armatures que l'on peut envisager emmanchées latéralement, donc recherchées avec des qualités tranchantes. Or, le grain des silex jaspéroïdes est particulièrement fin, favorisant le tranchant des produits issus de cette matière.

De plus, la modalité opératoire employée, unipolaire convergente, est adaptée à la production d'éléments proportionnellement larges et peu épais (Chalard et al. 2006 ; Guillermin 2004) (fig. 11a). Hormis les toutes premières phases de la chaîne opératoire, toute la séquence est présente sur le site jusqu'à l'utilisation et l'abandon de l'outil.

Les outils en silex bajocien sont essentiellement des outils à usage domestique. Cependant, ces derniers sont peu éla- borés (éclats retouchés, encoche, denticulé, burin sur cassure...) réalisés sur éclats, très souvent corticaux, issus des phases de mise en forme et d'entretien du débitage. Ces proportions ne sont pas révélatrices de l'exploitation effective de la matière la plus représentée au sein du débitage.

Cette exploitation est très différente de celle des jaspéroïdes. Tout d'abord, la matière a été introduite sur le site sous forme de rognons bruts, souvent de grand gabarit. La modalité opératoire employée est une exploitation frontale d'un volume au cintre resserré à l'aide d'un ou de deux plans de frappe (fig. 11b), favorisant l'épaisseur et la robustesse du support. Enfin, les supports produits sont absents de l'outillage, ils ont donc été exportés, sous forme brute ou retouchée. Compte tenu de leur rectitude, de leur gabarit laminaire et de leur robustesse, ils sont très différents des supports de lamelles à dos tronquées, se rapprochant de ceux des pointes de La Gravette.

II y a donc eu, pendant l'occupation du gisement, une exploitation adaptée des ressources locales qui, suivant leurs propriétés, remplissent des objectifs différents, avec des fractionnements différents des chaînes opératoires : des matières premières à grain très fin sont utilisées pour produire des supports minces particulièrement tranchants sur les bords, tandis que des matières premières plus grenues sont utilisées pour produire des supports plus robustes.

\subsection{2 - Polyvalence des ressources allochtones}

Les silex allochtones ont été introduits à différents stades de la chaîne opératoire : sous forme d'outils, de supports, de nucléus ou de blocs bruts. Les séquences de débitage présentes sur le site révèlent l'utilisation de différents schémas opératoires lamino-lamellaires (unipolaire convergent, exploitation d'un volume cintré à l'aide d'un ou de deux plans de frappe) ainsi que d'un débitage d'éclats. Ces différentes chaînes opératoires, pouvant se succéder dans l'exploitation d'un même bloc réorienté et débité jusqu'à exhaustion, sont révélateurs de la diversité des objectifs de production.

En outre, les supports d'outils en ces matières peuvent être replacés à tous les stades de la chaîne opératoire. Le cortex est bien représenté sur les supports d'outils à usage domestique (27\%) ainsi que sur les produits bruts (25\%), cela montre l'introduction de silex allochtones sous forme de rognons bruts (ou testés) ou de gros éclats corticaux. Ainsi, le transport de bloc brut permet, lors du débitage, la récupération sur place de sous-produits pour subvenir à différents types de besoins, traduisant ainsi une certaine économie du débitage (Perlès 1991).

\subsection{3 - Bilan}

Résumons à présent les données que nous avons sur les différents types de matière première :

Les silex jaspéroïdes ont fait l'objet d'une exploitation adaptée et orientée vers la production de lamelles à dos 

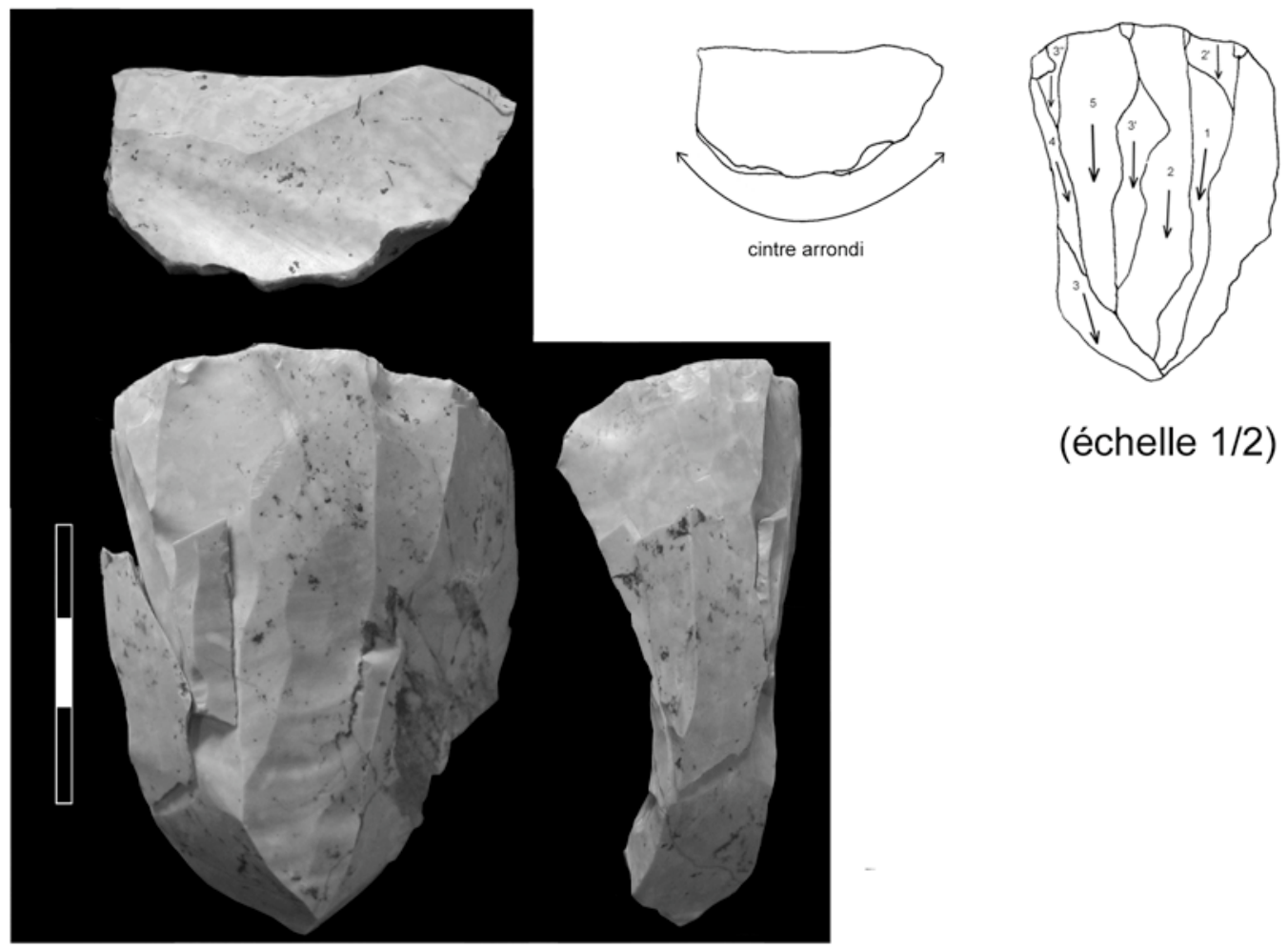

(échelle 1/2)
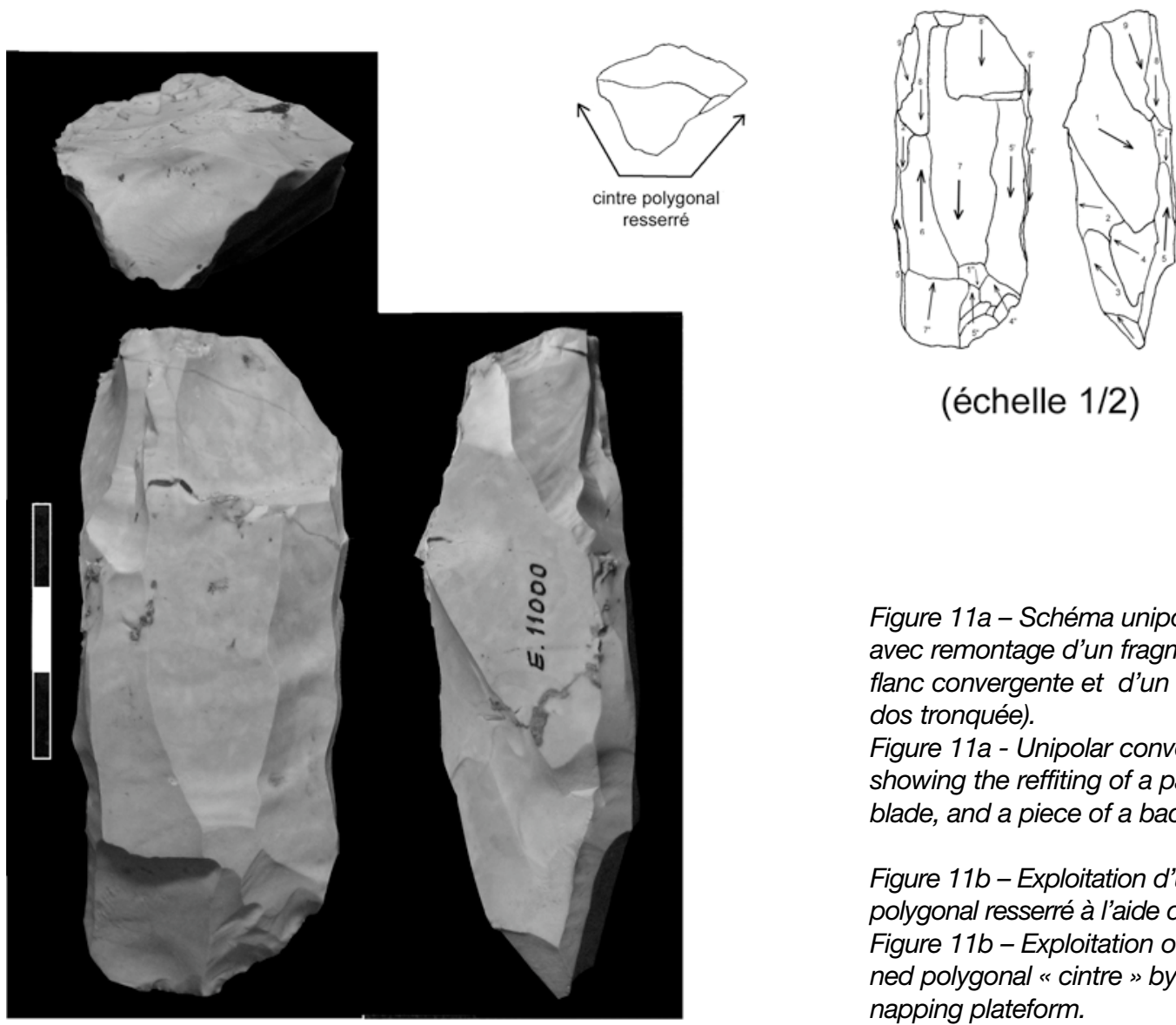

(échelle 1/2)

Figure 11a - Schéma unipolaire convergent (photo avec remontage d'un fragment distal de lame de flanc convergente et d'un fragment de lamelle à dos tronquée).

Figure 11a - Unipolar converging schema (picture showing the reffiting of a part of a side converging blade, and a piece of a backed truncated bladelet).

Figure $11 b$ - Exploitation d'un volume au cintre polygonal resserré à l'aide de deux plans de frappe. Figure $11 b$ - Exploitation of a volume with tightened polygonal " cintre » by the mean of a double napping plateform. 
tronquées. De plus, la présence dans l'assemblage de la totalité de la chaîne opératoire révèle un usage immédiat et ciblé. Ce phénomène est révélateur d'un investissement technique fort qui est à mettre en relation avec l'activité de chasse pratiquée aux abords du site.

Les silex allochtones du Sénonien ont, pour leur part, produit en grand nombre des outils à usage domestique. Ces derniers sont réalisés notamment à partir des sous-produits de la chaîne opératoire de production d'armatures. Ainsi, contrairement aux silex jaspéroïdes, les matières allochtones sont beaucoup plus polyvalentes. Elles peuvent être qualifiées de matière première de 'voyage', utilisées en réponse à l'ensemble des besoins que peut avoir le groupe durant ses déplacements, induisant un transport sous forme variée de la matière, du bloc brut à l'outil.

Ce stock diminuant, le groupe a exploité une matière locale adaptée pour la production de supports laminaires relativement robustes afin de restaurer le stock de supports, anticipant les besoins des futurs déplacements au sein du territoire.

Ainsi, sans parler de réelle économie des matières premières, des préférences se manifestent dans l'exploitation des ressources siliceuses. Elles révèlent une adaptation du groupe aux opportunités offertes par les ressources locales et un investissement technique raisonné pour répondre à des objectifs présents ou futurs, complété par l'anticipation que constitue l'apport de matière première de voyage. Tout ceci donne une image d'un groupe maîtrisant son parcours au sein d'un territoire dont l'étendue et le mode d'exploitation reste à préciser.

\section{4 - OUVERTURE SUR LA PROBLÉMATIQUE DE L'ÉTUDE DE L'OCCUPATION GRAVETTIENNE DU QUERCY}

\section{1 - Des assemblages lithiques résultant de l'interac- tion de différents types de contraintes}

Pour commenter la gestion des ressources siliceuses observée aux Fieux, citons J-M. Geneste : « [les stratégies économiques] peuvent être le résultat d'une cascade d'interactions entre trois types de contraintes : ressources $d u$ milieu, possibilités techniques au niveau des procédés et des méthodes, enfin besoins en produits finis " (Geneste 1991, p.16).

Les ressources du milieu ont été exploitées de manière adaptée à leurs propriétés. L'ensemble de la couche $\mathrm{E}$ illustre le lien pouvant exister entre la variabilité des schémas opérés, les différentes matières présentes dans l'environnement et le statut d'une production anticipée ou à usage immédiat.

Ainsi, concernant le besoin en produits finis, nous voyons le fort investissement technique qui a été mis en œuvre pour la production d'armatures, étayant l'hypothèse d'une spécialisation de l'occupation.
En développant l'idée de l'interaction entre les trois types de contraintes énoncées par J-M. Geneste, la question de leur hiérarchie se pose : dans le cas des Fieux, est-ce parce qu'ils cherchaient à produire des lamelles à dos tronquées que les paléolithiques ont ainsi exploité les silex jaspéroïdes ? Ou bien est-ce la présence de silex jaspéroïdes dans l'environnement de l'occupation qui a favorisé la production de lamelles à dos tronquées ?

Autrement dit, jusqu'où va la capacité d'adaptation du tailleur et par là-même la souplesse des Gravettiens ? La diversité des armatures ne peut-elle pas être le résultat d'une recherche de combinaison optimale des trois types de contraintes pour répondre à l'objectif d'une occupation spécialisée?

L'originalité de la couche $E$ soulève ainsi la question de la fonction du site et des implications d'une éventuelle spécialisation dans la constitution des assemblages. Aborder cette notion de spécialisation induit l'existence de sites complémentaires, qu'en est-il des autres occupations gravettiennes quercinoises?

II est difficile de répondre à cette question sans reprendre l'étude des séries. Aucun des sites ayant fourni des données satisfaisantes (La Bergerie, Les Battuts, Roc-deCombe), ne révèle, au travers des inventaires typologiques, des indices de spécialisation, à l'exception de l'Abri des Peyrugues. En effet, les armatures sont largement prédominantes au sein des ensembles gravettiens de ce gisement et les activités de débitage sont peu développées dans la plupart des cas. Ces éléments corroborent l'interprétation de l'occupation de ce site en tant que haltes de chasse (Allard 1996 ; Allard et al. 1997). Ce gisement, situé dans la vallée du Célé, au pied d'une falaise, a ainsi été l'objet d'occupation différente de celle des Fieux : les ressources siliceuses locales sont beaucoup moins exploitées et les outils à usage domestique sont très peu présents.

Ainsi, le bilan des données nous permet de constater que deux sites quercinois ont été témoins de l'importance de l'activité cynégétique au cours de leur occupation, mais de façon différente. Les données disponibles concernant les autres sites, ne permettent pas de caractériser une quelconque spécificité fonctionnelle.

\section{2 - De l'idée de spécialisation aux modèles d'occu- pation du territoire}

Aborder l'idée de la fonction des sites et donc de leur éventuelle spécialisation amène, par l'implication d'existence de sites complémentaires, à l'élaboration de modèles d'occupation du territoire. De grands axes privilégiés de trajectoire sont par ailleurs dessinés grâce à la caractérisation des ressources siliceuses. Ainsi, les études pétroarchéologiques des gisements des Fieux, des Peyrugues et de Pégourié révèlent une forte présence des matières premières périgourdines au sein des assemblages (Allard 1996 ; SéronieVivien 1995 ; Chalard et al. 2006). II existait donc une 'perméabilité' des territoires paléolithiques entre le Périgord et le Quercy. Cette constatation ouvre sur la problématique du 
statut éventuel du Quercy au sein d'un territoire de groupes gravettiens du Périgord. Contrairement à d'autres cultures (Allard 1996), aucune donnée de saisonnalité sur les niveaux gravettiens ne permet d'étayer l'hypothèse d'une occupation saisonnière de l'espace quercinois par les groupes concernés.

L'étude de la couche $\mathrm{E}$ des Fieux permet de dire que les occupants du site ont une partie de leur territoire en Périgord. lls sont venus chasser aux Fieux mais sont restés suffisamment longtemps pour exercer d'autres activités, abandonner une certaine quantité de leur matière première de voyage périgourdine et reconstituer une partie de leur équipement à l'aide des matières premières locales. Cette occupation était-elle l'une des principales motivations de leur déplacement ? Sont-ils en route vers le Massif central ?

\section{CONCLUSION}

Les études pétroarchéologiques révèlent un lien étroit entretenu avec le Périgord. II est cependant prématuré de tester des modèles d'occupation et d'exploitation de territoires englobant le Quercy, au sein desquels celui-ci pourrait avoir un statut particulier. II semble néanmoins que l'occupation gravettienne de cet espace ne peut être exclusivement assimilée à une occupation saisonnière limitée à la chasse : les sites d'art pariétal attribués au Gravettien sont en effet nombreux, témoignant d'un investissement symbolique important (Lorblanchet 1984a et b).

L'exemple de l'étude de la couche $\mathrm{E}$ des Fieux montre que le Quercy peut être un bon terrain d'investigation pour les préhistoriens cherchant à tester les capacités d'adaptation des groupes gravettiens, combinant les données environnementales et les différents objectifs - immédiats et différés - des étapes de parcours au sein du territoire.

\section{BIBLIOGRAPHIE}

ALAUX J.F. 1965 - Notes sur quelques pièces périgordiennes de la grotte du Rouset, commune de Larroque (Tarn). Travaux et recherches de la Fédération Tarnaise de Spéléo-Archéologie t. 3, p. 36-40, 1 pl.

ALAUX J.F. 1967 - Burins de type «Le Raysse» de l'abri des Battuts (Tarn). Bulletin de la Société Préhistorique Française t. 64, p. 242-246, 2 pl.

ALAUX J.F. 1969 - Note préliminaire sur l'abri périgordien des Battuts (Tarn). Bulletin de la Société Préhistorique Française t. 66, p. 10-15.

ALAUX J.F. 1973 - Pointes de La Font-Robert, en place, dans le Périgordien à burins de Noailles de l'abri des Battuts (commune de Penne, Tarn). Bulletin de la Fédération Tarnaise de Spéléologie et d'Archéologie t. 70 (2), p. 51-55, 2 fig.

ALLARD M. 1996 - Rapport de fouilles de l'Abri des Peyrugues. Synthèse des campagnes de 1994 à 1996. S.R.A Midi-Pyrénées.
ALLARD M., DRIEUX M., JARRY M., POMIES M.P. et RODIERE J. 1997 - Perles en bois du gisement des Peyrugues, à Orniac (Lot) ; Hypothèse sur l'origine du Protomagdalénien. Paléo t. 9, p. 355-369.

BORDES F. 1968 - La question Périgordienne, La Préhistoire, problèmes et tendances, Editions du CNRS, p. 59-70, 3 fig.

BORDES F. 1978 - Le Protomagdalénien de Laugerie-Haute Est (fouilles F.Bordes). Bulletin de la Société Préhistorique Française t. 75 (11-12), p. 501-521, 11 fig., 2 tab.

BORDES F. et LABROT J. 1967 - La stratigraphie du gisement de Roc-de-Combe (Lot) et ses implications. Bulletin de la Société Préhistorique Française t. 64 (1), p. 15-28, 6 fig.

BORDES F. et SONNEVILLE-BORDES D. (de) 1966 Protomagdalénien ou Périgordien VII ? L'Anthropologie t. 70 (1-2), p. 113-122, 5 fig.

BRICKER H.M. 1995 - Le paléolithique supérieur de l'abri Pataud (Les Eyzies, Dordogne). Les fouilles de H.L. Movius $J r$, Documents d'Archéologie Française, Maison des Sciences de l'Homme. 328 p.

CHALARD P., GUILLERMIN P. et JARRY M. 2006 Acquisition et exploitation des silex allochtones au Gravettien : l'exemple de la couche E des Fieux (Lot, France). In : BRESSY C., BURKE A., CHALAND P. et MARTIN H. (dir) : "Notions de territoire et de mobilité en Préhistoire : exemple de l'Europe et des premières nations en Amérique du Nord avant le contact européen", actes de session au $X^{e}$ Congrès du Colloque de The European Association of Archaeologists, Lyon, 8-12 septembre 2004, ERAUL 116, p. 29-40, 10 fig., 2 tabl.

CHAMPAGNE F. 1977 - Essai d'interprétation chronologique des niveaux supérieurs de la grotte des Fieux, à Miers, Lot. Pré-publication du colloque de Bordeaux, Compléments p. 16, 5 fig.

CHAMPAGNE F. 1987 - Aurignacien et Périgordien dans le Haut-Quercy: l'apport des Fieux. Antiquités Nationales t.18-19, p. 63-68, 1 tab.

CHAMPAGNE F. et JAUBERT J. 1979 - La grotte des Fieux à Miers (Lot). Bilan de 13 années de recherche. XXI ème Congrès préhistorique de France, Montauban-Cahors. Vol. 1, p. 85-104, 13 fig.

CHAMPAGNE F. et JAUBERT J. 1986 - Un exemple de remplissage archéologique en milieu karstique: la grotte des Fieux, à Miers (Lot). Bulletin de la Société Méridionale de Spéléologie et de Préhistoire t. XXVI, p.21-33, 3 fig.

CHAMPAGNE F., CHAMPAGNE C., JAUZON P. et NOVEL P. 1990 - Le site des Fieux. Gallia Préhistoire. t. 32 (1), p.1-28.

CHAMPAGNE F., CHAMPAGNE DR C. et NOVEL P. 1996 Le site des Fieux dans les causses du Quercy. Relations 
entre l'homme préhistorique et son milieu, La vie préhistorique, Société Préhistorique Française. p.150-155.

CLAY R. B. 1968 - The protomagdalénian culture. Unpublished Ph.D., dissertation presented to the Dept. of Anthropology, Southern Illinois University. 2 vol. 660 p. 82 fig. 198 tab.

CLOTTES J. 1971 - Le Lot préhistorique (Additions et corrections). Bulletin de la Société des Études Littéraires, Scientifiques et Artistiques du Lot, t. suppl. au $1^{\text {er }}$ fasc., janvier-mars, p. 287-302, 8 fig.

CLOTTES J. 1979 - Informations archéologiques. Gallia Préhistoire t. 22, fasc. 2.

CLOTTES J., LORBLANCHET M., FAU M.F. et PEYRE G. 1990 - L'abri périgordien de la Bergerie, à Saint-Géry (Lot). Bulletin de la Société Préhisrtorique Française t. 87 (10-12), p.342-357, 15 fig. 3 tab.

COULONGES L. 1949 - Le gisement paléolithique de Cavart, Lot, commune de Montcabrier. L'Anthropologie t.53, p.558-560, 2 fig.

DELPORTE H. 1954 - Le Périgordien. Bulletin de la Société Préhistorique Française t. 51, p. 44-48.

DELPORTE H. 1972 - Protomagdalénien du Blot, commune de Cerzat (Haute-Loire). Congrès Préhistorique de France, XIX session, Auvergne, 1972 p. 190-199, 7 fig.

DELPORTE H. 1983 - L'organisation du Périgordien Supérieur en France et ses rapports avec le Périgordien d'Europe Occidentale. Etudes et recherches Archéologiques de I'Université de Liège, t. 13 (1), p.83-106.

GENESTE J.M. 1991 - Systèmes techniques de production lithique: variations techno-économiques dans les processus de réalisation des outillages paléolithiques. Techniques et cultures t. 17-18, p. 1-35, 5 fig.

GLORY A. 1965 - Nouvelles découvertes de dessins rupestres sur le Causse de Gramat (Lot). Bulletin de la Société Préhistorique Française, t. 62, Études et Travaux 3 , p. 528-538, 10 fig.

GUILLERMIN P. 2003 - Etude technologique d'un assemblage lithique extrait d'une collection de ramassage de surface: l'atelier du Ségalar (commune du Verdier, Tarn). Hypothèse d'attribution chrono-culturelle au Gravettien à partir d'une comparaison avec la couche 5 de l'abri des Battuts (commune de Penne, Tarn). Mémoire de Maîtrise. Université Toulouse Le Mirail, $177 \mathrm{p}$.

GUILLERMIN P. 2004 - Réflexions sur l'interprétation des industries gravettiennes à partir de l'étude techno-typologique d'une occupation spécialisée: la couche $E$ du gisement des Fieux (Miers, Lot). Mémoire de DEA d'Anthropologie, 99 p., 48 fig., 3 tab.
GUILLERMIN P. 2006 - Les Fieux : une occupation gravettienne du causse quercinois. Paleo, $n^{\circ} 18$, p. 69- 94, 15 fig., 4 tab.

GUILLERMIN P. et MORALA A. à paraître - " Les "Périgordiens » étaient-ils quercinois ? ». In : Modalités d'occupations et exploitation des milieux au Paléolithique dans le Sud-Ouest de la France : l'exemple du Quercy. Actes du Colloque organisé dans le cadre du XVI ${ }^{\text {e }}$ Congrès de I'UISPP, JARRY M. (dir.), BRUGAL J.-P. (dir.), FERRIER C. (dir.), Lisbonne, sept. 2006, suppl. Paléo.

KLARIC L. 2003 - L'unité technique des industries à burins du Raysse dans leur contexte diachronique. Réflexions sur la diversité culturelle au Gravettien à partir des données de la Picardie, d'Arcy-sur-Cure, de Brassempouy et du Cirque de la Patrie. Thèse de Doctorat de l'Université de Paris I. 429 p.

KLARIC L., AUBRY T. et WALTER B. 2002 - Un nouveau type d'armature en contexte gravettien et son mode de production sur les burins du Raysse (la Picardie, commune de Preuilly-sur-Claise). Bulletin de la Société Préhistorique Française t.99 (4), p. 751-764.

LAVILLE H. et RIGAUD J.-PH. 1973 - The perigordian V industries in Perigord: typological variations, stratigraphy and relative chronology. World Archaeology t.4 (3). p. 330338, 2 fig., 2 tab.

LE TENSORER J.M. 1981 - Le Paléolithique en Agenais, Cahiers du Quaternaire. $n^{\circ}$ 3, p. 526, 212 fig., 55 tab.

LORBLANCHET M. 1966 - Sondage dans la grotte des Escabasses, commune de Thémines (Lot). Bulletin de la Société Préhistorique Française t. 63, Études et Travaux fasc. 2, p. 262-278, 5 fig.

LORBLANCHET M. 1984a - L'art des cavernes. Atlas des grottes ornées paléolithiques françaises. Ministère de la Culture, Imp. nat., Paris.

LORBLANCHET M. 1984b - Nouvelles découvertes d'art pariétal en Quercy. L'art pariétal paléolithique; Périgueux. Le Thot, 1984, Ministère de la culture, p. 79-105.

LORBLANCHET M. ET GENOT L. 1972 - Quatre années de recherches préhistoriques dans le Haut-Quercy. Bulletin de la Société des Études Littéraires, Scientifiques et Artistiques du Lot t. 93 (2), p. 71-153, 42 fig.

LORIN Y. 1996 - Rabier (commune de LanquaisDordogne), un site de plein air du Bergeracois. Un outillage du Périgordien évolué au fil du temps. Mémoire de maîtrise Université de Paris I. 67 p.

LUCAS G. 2000 - Les industries lithiques du Flageolet (Dordogne) approche économique, technologique, fonctionnelle et spatiale. Thèse de doctorat de Préhistoire et Géologie du Quaternaire de I'Université de Bordeaux I. 2 vol., $600 \mathrm{p}$. 
MORALA A. 1984 - Périgordien et Aurignacien en HautAgenais. Etudes d'ensembles lithiques. Archives d'Ecologie Préhistorique, Ecole des Hautes Etudes en Sciences Sociales, Toulouse, Mémoire 7, 140 p., 42 fig.

MORALA A. 1989 - Les voies de communication au Paléolithique supérieur en Aquitaine du Nord. L'exemple du Haut-Agenais. Paléo t.1., p.31-35, 1 fig.

MORALA A. 1990 - L'atelier du Périgordien supérieur de Rabier (Lanquais, Dordogne): recherche sur l'origine des occupants du site sur les bases de la lithologie. in: Le silex de sa genèse à l'outil, Actes du Vème Colloque international sur le silex, 1990. Vol. 17, Cahiers du Quaternaire, p. 391-404.

NOUGIER L.-R. et BARRIERE C. 1965 - La nouvelle grotte préhistorique des Fieux et ses mains négatives. $L a$ Nature t. 3359, p. 81-86.

PAJOT B. 1969 - Les civilisations du Paléolithique supérieur du bassin de l'Aveyron, Travaux de l'Institut d'Art Préhistorique 11., p. 584.

PERLES C. 1991 - Economie des matières premières et économie du débitage: deux conceptions opposées? In : $X l^{\ominus}$ rencontre internationale d'Archéologie d'Antibes. 25 ans d'études technologiques en Préhistoire: bilan et perspectives., Actes des rencontres 18-19-20 octobre 1990. Juan-les-Pins : APDCA, 1991 p. 35-45.

PEYRE G. 1988 - L'Abri de la Bergerie, à Saint-Géry (Lot). Toulouse, Ecole des Hautes Etudes en Sciences Sociales, 1988,127 , p. 61 , fig.

PEYRONY D. 1946 - Une mise au point au sujet de l'Aurignacien et du Périgordien. Bulletin de la Société Préhistorique Française t.43 (7), p. 232-237.
POTTIER C. 2005 - Le Gravettien moyen de l'abri Pataud (Dordogne, France): le niveau 4 et l'éboulis 3/4. Etude technologique et typologique de l'industrie lithique. Mémoire de doctorat du Museum d'Histoire Naturelle, 396 p.

RIGAUD J.-Ph. 1982 - Le Paléolithique en Périgord: les données du Sud-Ouest Sarladais et leurs implications. Thèse de doctorat d'état de l'Université de Bordeaux I. 2 vol. 294, p. 242 fig. 17, tab.

RIGAUD J.-Ph. 1983 - Données nouvelles sur l'Aurignacien et le Périgordien en Périgord. Etudes et recherches Archéologiques de I'Université de Liège, ERAUL t. 13 (1), p.107-118, 2 fig.

RIGAUD J.-Ph. 1985 - Réflexions sur la signification de la variabilité des industries lithiques paléolithiques. Colloque "La signification culturelle des industries lithiques», Liège, 1985, Vol. 239 British Archaeological Report International p. $374-380$.

SACKETT J. 1999 - The archaeology of Solvieux: an upper open air site in France., Monumenta Archaeologia, Institute of Archaeology, University of California. 19.

SERONIE-VIVIEN M.R. 1995 - La grotte de Pégourié. Caniac-du-causse (Lot). Préhistoire Quercinoise. Supplément $n^{\circ} 2$, p. 334 .

SONNEVILLE-BORDES D. (de) 1966 - L'évolution du Paléolithique Supérieur en Europe occidentale et sa signification. Bulletin de la Société Préhistorique Française t. 63 (3-34).

SONNEVILLE-BORDES D. (de) 2002 - Les industries de Roc-de-Combe (Lot). Périgordien et Aurignacien. Bulletin de Préhistoire du Sud-Ouest t. 9, p. 121-161, 29 fig., 2 tab. 